\title{
Efficient Calibration Tool for Thermistor
}

\author{
Jong-Dae Kim ${ }^{1,2}$, Doo-Hee Jeong ${ }^{3}$, Hye-Jeong Song ${ }^{1,2}$, \\ Yu-Seop Kim ${ }^{1,2}$ and Chan-Young Park ${ }^{1,2, *}$ \\ 'Department of Convergence Software, Hallym University, \\ Chuncheon, Ganwondo 200-702, Korea \\ ${ }^{2}$ Bio-IT Research Center, Hallym University, Chuncheon, Ganwondo 200-702, Korea \\ ${ }^{3}$ Department of Computer Engineering, Hallym University, \\ Chuncheon, Ganwondo 200-702, Korea
}

(Received July 2, 2014; accepted February 9, 2015)

Key words: thermistor, temperature coefficient, Steinhart-Hart equation, calibration

We propose a tool for calibrating the coefficient variables of the Steinhart-Hart equation, which are used in temperature measurement with a negative-temperaturecoefficient thermistor. The previous method modifies the coefficient variables manually, but the proposed tool takes a measured temperature and automatically modifies the coefficient variables. The proposed calibration tool provides a graphical user interface program for the convenience of users. It is applied to the 4-point temperature measurement of polymerase chain reaction and has a degree of precision of $\pm 0.1^{\circ} \mathrm{C}$ in the temperature measurement evaluation.

\section{Introduction}

For temperature measurement within a limited range, a negative-temperaturecoefficient (NTC) thermistor ${ }^{(1)}$ has many advantages, such as high sensitivity, fast thermal response, availability of various shapes and sizes, and low cost; thus, owing to these advantages, the thermistor is preferred over other equipment. ${ }^{(2,3)}$ The thermistor is an element that measures temperature. Its measured resistance can be changed to the current thermistor temperature. The relationship between the resistance and temperature of the thermistor is nonlinear, and it is nearly exponential. The relationship is accurately analyzed using the Steinhart-Hart $(\mathrm{S}-\mathrm{H})$ equation: ${ }^{(4)}$

$$
T^{-1}=A+B \cdot \ln R+C \cdot(\ln R)^{3},
$$

where $A, B$, and $C$ are the dimensionless parameters called the $\mathrm{S}-\mathrm{H}$ coefficients, and $R$ is the measured resistance of the thermistor. This formula can generally achieve a degree of precision of $\pm 0.01{ }^{\circ} \mathrm{C}$ within the range from 0 to $100{ }^{\circ} \mathrm{C}$. Manufacturers normally

"Corresponding author: e-mail: cypark@hallym.ac.kr 
provide the S-H coefficients of thermistors, and thermistors of the same model usually have the same values. However, these coefficients can be different for each thermistor. Thus, for more exact temperature measurements with each thermistor, there is a need to modify the S-H coefficients slightly. ${ }^{(5)}$

The process for modifying the S-H coefficients of a thermistor is called calibration. The previous modification process ${ }^{(6)}$ required a known temperature and the measured resistance to adjust the coefficient variables manually;(7) however, the proposed calibration tool automatically adjusts the basic S-H coefficients. This tool is userfriendly in the sense that it simply records the difference between the measured temperature and the known temperature in a graphic user interface (GUI) program. Thus, it is convenient for users. It is applied to the 4-point temperature measurement of polymerase chain reaction and has a degree of precision of $\pm 0.1{ }^{\circ} \mathrm{C}$ in the temperature measurement evaluation.

\section{Materials and Methods}

\subsection{Proposed calibration tool}

The S-H coefficients before and after the modification are marked $A, B$, and $C$, and $A^{\prime}, B^{\prime}$, and $C^{\prime}$, respectively. The temperature measured using the basic $\mathrm{S}-\mathrm{H}$ coefficient is marked $T$. The known basic temperature in a stable liquid bath is marked $T^{R}$. When measuring the basic temperature in a liquid bath, if the basic S-H coefficients are not the same, $T$ will be different from the accurate value of $T^{R}$. The proposed calibration tool changes the basic S-H coefficients so that $T=T^{R}$.

The tool first uses the following eq. (2) to calculate the resistance $R$ of the thermistor from $T$ and the basic S-H coefficients $(A, B$, and $C$ ). Equation (2) is the inverse of eq. (1).

$$
R=\exp (\sqrt[3]{\beta-\alpha}-\sqrt[3]{\beta+\alpha}), \text { where } \alpha=\frac{A-\frac{1}{T}}{2 C} \text { and } \beta=\sqrt{\left(\frac{B}{3 C}\right)^{3}+\alpha^{2}}
$$

Next, the tool applies $R$ and $T^{R}$ to eq. (1) to calculate the S-H coefficients $A^{\prime}, B^{\prime}$, and $C^{\prime}$. For each resistance $\left(R_{1}, R_{2}\right.$, and $\left.R_{3}\right)$ calculated at the known basic temperature, three modified coefficient variables $\left(A^{\prime}, B^{\prime}\right.$, and $\left.C^{\prime}\right)$ can be determined by inserting the three resistances and the three known temperatures into eq. (3).

$$
\frac{1}{T_{i}^{R}}=A^{\prime}+B^{\prime} \cdot \ln R_{i}+C^{\prime} \cdot\left(\ln R_{i}\right)^{3} \text { for } i=1,2,3
$$

The proposed calibration tool provides a GUI program as shown in Fig. 1. The GUI operates under Windows XP or Windows 7, and it was developed using Microsoft's Visual Studio 2008 and Microsoft Foundation Class (MFC). In this screen shot, the serial number in the edit box (left topmost) indicates the set ID to be calibrated, and the following $4 \times 4$ boxes present calibration data from the sensors at each reference temperature of $50,60,72$, and $95^{\circ} \mathrm{C}$. 


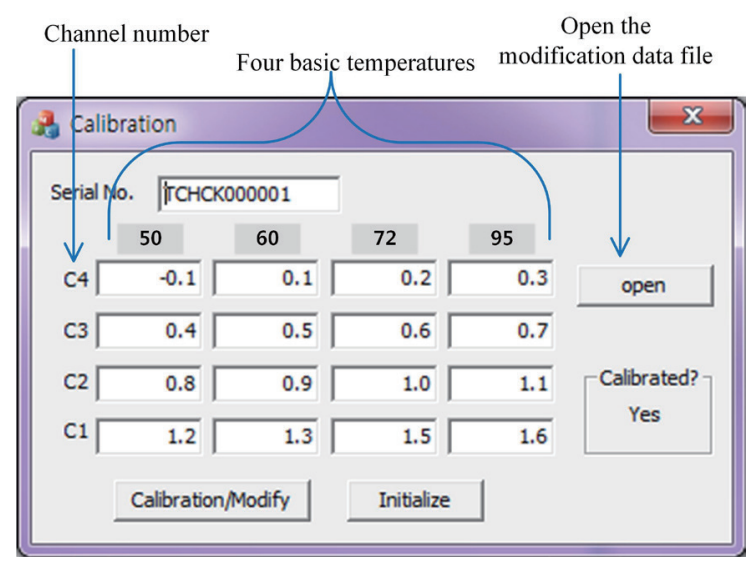

Fig. 1. (Color online) Calibration tool.

For simple calibration handling and a one-step calibration process, users only record the difference between the measured temperatures and the basic temperature as the entered parameter in the GUI program. For example, if at $50{ }^{\circ} \mathrm{C}$, as in sensor 1 in Fig. 1 , the thermistor reads $51.2{ }^{\circ} \mathrm{C}$, exhibiting a difference of $+1.2^{\circ} \mathrm{C}$, then the edit box in the 1 st column of the 4 th row of the $4 \times 4$ edit boxes will show 1.2. The $4 \times 4$ edit boxes can be filled by reading the file using the 'Open' button. Next, the tool calculates the measured temperature $T$ from the entry parameter. With the given temperature $T$ and the basic S-H coefficients, this tool uses eq. (2) to deduce the resistance $R$. The deduced resistance $R$ and the basic temperature $T^{R}$ are applied to eq. (3) to determine the modified S-H coefficients $A^{\prime}, B^{\prime}$, and $C^{\prime}$.

Figure 2 shows a tool for displaying the current temperatures of the thermistors. This tool also shows the maximum, minimum, average and current temperatures of four sensors of two channels. It has a record function for further analysis of the temperature behaviors of the sensors.

Figure 3 shows the four-point temperature measurement system for verifying the thermal cyclers for polymerase chain reaction (PCR), whose sensors should be calibrated. It has PIC18F4458 (Microchip Technology Inc.) as the main controller and is composed of two channels. Two sensors can be attached to each channel; thus, we should calibrate four sensors at one time. To obtain four reference temperatures, a constant water bath from Heto was employed and the temperature is measured with a laboratory-grade temperature probe with $0.01{ }^{\circ} \mathrm{C}$ accuracy (U.S. Sensor Corporation). After measuring the resistance of the probe using a USB digital multimeter (USB-4065, National Instruments Corporation), the thermistor datasheet from U.S. Sensor Corporation was used as reference to obtain the exact temperature of the water bath.

Figure 4 shows another tool, which can be used to navigate all of the calibrated sets. The modification data and the modified S-H coefficients are in a specific data file, and the entire modified set list in the list box appears when reading the file by pressing the 


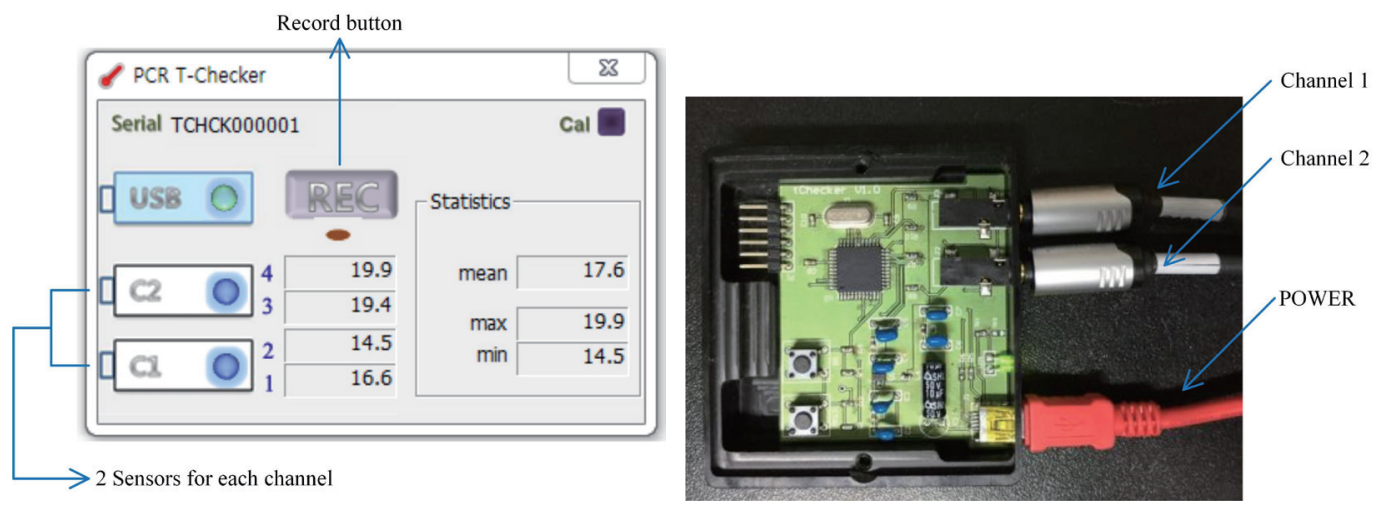

Fig. 2 (left). (Color online) Temperature measurement tool.

Fig. 3 (right). (Color online) Four-point temperature measurement system for thermal cycler.

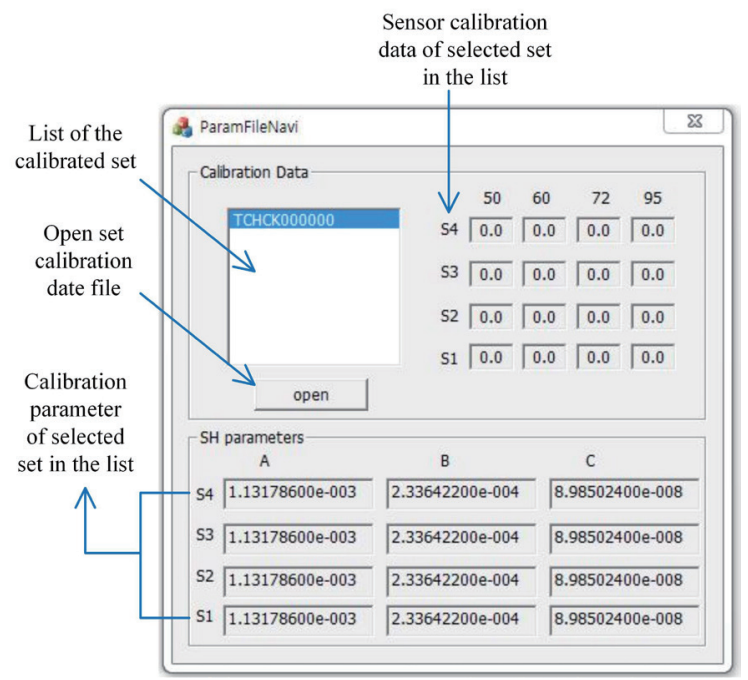

Fig. 4. (Color online) Tool showing all calibrated sets.

'Open' button. When selecting a set in the list box, the temperature differences used in the modification appear in the $4 \times 4$ edit boxes on the upper right part, and the S-H coefficients used for this are listed in the $4 \times 3 \mathrm{SH}$ parameter boxes on the lower part. 


\subsection{Calibration accuracy evaluation}

The proposed tool uses four different temperatures, namely, 50, 60, 72, and $95{ }^{\circ} \mathrm{C}$, which are important temperatures for PCR. ${ }^{(8)}$ For the modification of each channel at the four temperature points, we applied the least-squares optimization method to minimize the sum of the measurement errors.

First, we can obtain four linear equations for the three unknown S-H coefficients $A^{\prime}, B^{\prime}$, and $C^{\prime}$, applying the four known temperatures $T_{1}^{R}, T_{2}^{R}, T_{3}^{R}$, and $T_{4}^{R}$, and their corresponding resistances $R_{1}, R_{2}, R_{3}$, and $R_{4}$.

$$
\frac{1}{T_{i}^{R}}=A^{\prime}+B^{\prime} \cdot \ln R_{i}+C^{\prime} \cdot \ln R_{i} \text { for } i=1,2,3,4
$$

The above overdetermined system of the linear equations can be solved using the pseudoinverse of the system matrix, yielding the least-squares error. This was implemented in the proposed tool to modify the basic S-H coefficients using the pseudoinverse.

To evaluate the accuracy of temperature measurement, we used 16 thermistors from U.S. Sensor Corporation, all of which had an error range of $\pm 0.4{ }^{\circ} \mathrm{C}$ using the same basic $\mathrm{S}-\mathrm{H}$ coefficients. The temperature measurement accuracies before and after applying the calibration tool were then compared.

\section{Results}

Table 1 shows the temperature errors before and after the calibration. In the temperature measurements using the basic $\mathrm{SH}$ coefficients, the maximum measurement error was $0.4{ }^{\circ} \mathrm{C}$, and the average measurement error was $0.11-0.25^{\circ} \mathrm{C}$.

In the temperature measurements using the modified $\mathrm{SH}$ coefficients after the calibration, the maximum measurement error was $0.1{ }^{\circ} \mathrm{C}$ and the average measurement error was $0.01-0.06^{\circ} \mathrm{C}$.

We also compared the theoretical error distributions of the measured temperatures according to the temperature before and after. The convex curve resulted from the basic $\mathrm{S}-\mathrm{H}$ coefficients, and the nearly straight line, from the calibrated S-H coefficients. This shows that we can achieve a uniform precision of less than $\pm 0.1{ }^{\circ} \mathrm{C}$ error over the entire temperature range when we use the proposed calibration tool.

Table 1

Maximum and mean temperature errors before and after calibration $\left({ }^{\circ} \mathrm{C}\right)$.

\begin{tabular}{llllll}
\hline Reference temperature & & 50 & 60 & 72 & 95 \\
\hline \multirow{2}{*}{ Before calibration } & Max & 0.4 & 0.2 & 0.4 & 0.2 \\
\cline { 2 - 6 } & Mean & 0.25 & 0.14 & 0.15 & 0.11 \\
\hline \multirow{2}{*}{ After calibration } & Max & 0.1 & 0.1 & 0.1 & 0.1 \\
\cline { 2 - 6 } & Mean & 0.03 & 0.01 & 0.06 & 0.05 \\
\hline
\end{tabular}




\section{Conclusions}

We proposed a calibration tool that automatically adjusts the basic S-H coefficients for a thermistor. The proposed calibration tool simply records the difference between a measured temperature and the basic temperature, which is highly convenient for users. The proposed tool can be applied to the four-point temperature measurement of PCR. In the temperature measurement evaluation of 16 thermistors, the proposed calibration tool improved their precision to $\pm 0.1{ }^{\circ} \mathrm{C}$, which considerably increased their temperature measurement accuracy.

\section{Acknowledgements}

This research was supported by the Basic Science Research Program through the National Research Foundation of Korea (NRF) funded by the Ministry of Education (2013R1A1A2013490).

\section{References}

1 C. Chen: Measurement 42 (2009) 1103.

2 H.-G. Schweiger, M. Multerer and H. J. Gores: IEEE T. Instrum. Meas. 56 (2007) 2002.

3 V. Maric, M. D. Lukovic, L. Zivanov, O. Aleksic and A. Menicanin: IEEE T. Instrum. Meas. 57 (2008) 2568.

4 J. S. Steinhart and S. R. Hart: Deep-Sea Res. 15 (1968) 497.

5 J. D. Kim, C. Y. Park, J. M. Kim, Y. S. Kim and H. J. Song: Int. J. Control Autom. 6 (2013) 417.

6 J. M. Kim, C. Y. Park, Y. S. Kim, H. J. Song and J. D. Kim: Int. J. Control Autom. 6 (2013) 413.

7 M. D. Alexander and K. T. B. MacQuarrie: Ground Water Monit. R 25 (2005) 75.

8 T. A. Brown: Gene Cloning and DNA Analysis: An Introduction (Wiley-Blackwell Publishing, West Sussex, 2010) Chap. 9. 